\title{
Carriage of Streptococcus Pneumoniae In Unvaccinated Toddlers At The Time of Pneumococcal Conjugate Vaccine Implementation Into The National Immunization Program In Poland
}

Izabela Wróbel-Pawelczyk

Department of Epidemiology and Clinical Microbiology, National Medicines Institute, Warsaw

\section{Patrycja Ronkiewicz}

Department of Epidemiology and Clinical Microbiology, National Medicines Institute, Warsaw

Monika Wanke-Rytt

Department of Pediatrics with Clinical Assessment Unit, Medical University of Warsaw, Warsaw

\section{Dominika Rykowska}

Department of Pediatrics with Clinical Assessment Unit, Medical University of Warsaw, Warsaw

\section{Aneta Górska-Kot}

Niekłańska Children's Hospital, Pediatrics, Warsaw

\section{Katarzyna Włodkowska}

Medical Centre MEDICERS Zacisze, Warsaw

\section{Agnieszka Topczewska-Cabanek}

Department of Family Medicine, Medical University of Warsaw, Warsaw

\section{Teresa Jackowska}

The Centre of Postgraduate Medical Education, Department of Pediatrics, Warsaw

\section{Joanna Chruszcz}

Department of Pediatric Infectious Diseases, Wroclaw Medical University, Wrocław

Walentyna Marchut

Białołęka Medical Centre, Warsaw

\section{Agnieszka Mastalerz-Migas}

Department of Family Medicine, Wroclaw Medical University, Wrocław

\section{Krzysztof Korzeniewski}

Department of Epidemiology and Tropical Medicine, Military Institute of Medicine, Warsaw

Anna Skoczyńska ( $\square$ a.skoczynska@nil.gov.pl)

Department of Epidemiology and Clinical Microbiology, National Medicines Institute, Warsaw

\section{Krzysztof Trzciński}

Department of Pediatric Immunology and Infectious Diseases, Wilhelmina Children's Hospital, University Medical Center Utrecht, Utrecht

\section{Research Article}

Keywords: polysaccharide vaccine, national immunization program, serotypes, surveillance 
DOI: https://doi.org/10.21203/rs.3.rs-810749/v1

License: (c) This work is licensed under a Creative Commons Attribution 4.0 International License. Read Full License 


\section{Abstract}

We investigated pneumococcal carriage among unvaccinated children under five years of age at the time of conjugate polysaccharide vaccine (PCV) introduction into the national immunization program (NIP). Paired nasopharyngeal swab (NPS) and saliva samples collected between 2016 and 2020 from n=394 children were tested with conventional culture and using qPCR. The carriage rate detected by culture was $25.4 \%$ (97 of 394), by qPCR $39.1 \%$ (155 of 394 ), and $40.1 \%$ (158 of 394) overall. The risk of carriage was significantly elevated among day care center attendees, and during autumn/winter months. Among strains cultured, the most common serotypes were: 23A, 6B, 15BC, 10A, 11A. The coverage of PCV10 and PCV13 was 23.2\% (23 of 99) and 26.3\% (26 of 99), respectively. Application of qPCR lead to detection of 168 serotype carriage events, with serogroups $15,6,9$ and serotype 23 A most commonly detected. Although the highest number of carriers was identified by testing NPS with qPCR, saliva significantly contributed to the overall number of detected carriers. Co-carriage of multiple serotypes was detected in $25.3 \%$ (40 of 158 ) of carriers. Results of this study represent a baseline for the future surveillance of effects of pneumococcal vaccines in NIP in Poland.

\section{Introduction}

Streptococcus pneumoniae is the common cause of invasive bacterial disease [1, 2]. Incidence of invasive pneumococcal disease (IPD) is highest among infants and toddlers and in older adults [1]. Despite available vaccines, in 2015 pneumococcus was responsible globally for approximately 300000 deaths of children under 5 years of age [2]. IPD is manifested by meningitis, sepsis and/or bacteremic pneumonia. S. pneumoniae also causes milder infections manifested across all ages as sinusitis or non-bacteremic pneumonia, and in children as acute otitis media. The primary virulence factor of pneumococci is the polysaccharide capsule [3] and currently available pneumococcal vaccines are all based on capsular polysaccharides as antigen. While there have been almost 100 capsular types (serotypes) described [4], marketed vaccines target only a subset of ten to twenty-three serotypes (24 in total). Pneumococcal conjugate vaccines (PCV) recommended for children [2], target ten (PCV10) and thirteen (PCV13) serotypes common in paediatric disease prior to PCVs introduction. PCV10 and PCV13 have been commercially available in Poland since 2009 and 2010, respectively. In 2017, PCV10 was introduced into the National Immunization Program (NIP) for all children born after 31st of December 2016, and PCV10 was chosen as the refunded vaccine in all consecutive annual NIP tenders. Children are vaccinated at 2, 4, and 13-15 month of life ( $2+1$ schedule). However, it is estimated that a quarter to a third of infants in Poland is vaccinated with PCV13 outside NIP [5].

Three years after PCV10 implementation into NIP, there was a significant decrease of IPD cases caused in Poland by PCV10 vaccine serotypes (VT) in children under 2 years of age (57\% in years $2014-2016$ vs. $31 \%$ in years 2017-2019) [6]. There was also a significant decline from 35-28\% in PCV10-VTs IPDs in persons $\geq 65$ years old. Similar herd effects were earlier observed in other countries [7-9]. Indirect effects are attributed to PCVs preventing VT strains carriage acquisition in vaccinees $[10,11]$. Since children are the main reservoir and the main transmitters of pneumococci, infants' vaccination with PCVs may have impact on carriage and disease in unvaccinated individuals in the same population $[3,12,2]$. Consequently, effects of PCVs can be monitored not only via surveillance of disease but also of carriage [13].

Since 1997 the National Reference Centre for Bacterial Meningitis (NRCBM) collects isolates causing IPD from the whole country and also conducts molecular diagnostics of IPD cases. Although surveillance of IPD in Poland is well established, the data on S. pneumoniae and pneumococcal serotypes carriage is limited. Over the past twenty years there have been only two studies published on pneumococcal carriage conducted in Poland [14-17]. To fill the gap, we investigated the pneumococcal carriage in unvaccinated children under five years of age. Our goal was to map the 
carriage of $S$. pneumoniae serotypes in paediatric population before the nationwide immunization of infants with PCV10 may result in substantial indirect effects. With this, we were aiming to establish a baseline for the future surveillance studies.

The gold standard for pneumococcal carriage detection is isolation of live $S$. pneumoniae from a culture of a deep trans-nasal nasopharyngeal swab (NPS) $[18,19]$. There is evidence that other samples, oral fluids in particular, may substitute for NPS [20,21]. Compared to NPS, saliva is much easier to collect, can be even self-collected, and, except for the youngest children, it does not require a designated collection kit. It has been also reported that application of molecular methods allows higher sensitivity of $S$. pneumoniae and pneumococcal serotypes carriage detection [2224]. Hence, our second goal was to compare results of carriage detection by testing saliva versus NPS, and using molecular methods versus conventional diagnostic culture in order to develop a procedure tailored to our future studies [25-27]. To our knowledge, there are no published studies comparing saliva testing with the gold standard in toddlers.

\section{Results}

Altogether 405 children have been enrolled in the study. Of these, nine children were excluded from further analysis for either being too young $(n=4)$, too old $(n=4)$, or being diagnosed with lower respiratory tract infection on enrolment day $(n=1)$. Two children were enrolled twice in the study and results of the first sampling were the only included. We report results for paired NPS and saliva collected once from 394 children.

\section{Study population}

Frequency of inclusions declined over the study years (Table 1) with half of children enrolled by the 14th month (September 2017) of 44-months long project. The number of 12-23-months old children $(n=147$ of 394) was significantly higher compare with any other age group (Fisher Exact, $p<0.001$ ), and the number of 24-35 months old $(n=102)$ was significantly higher compare to $36-47$ months and $48-59$ months olds $(n=71$ and $n=74$, respectively, $p<0.01)$. 
Table 1

Effect of study period, child's age, gender and environmental factors on S. pneumoniae carriage detected using culture and qPCR-based method.

\begin{tabular}{|c|c|c|c|c|c|c|}
\hline \multirow[t]{4}{*}{ attribute } & \multirow{4}{*}{$\begin{array}{l}\text { number of } \\
\text { individuals }\end{array}$} & \multicolumn{5}{|c|}{ carriers detected with: } \\
\hline & & \multicolumn{2}{|c|}{ conventional culture } & \multicolumn{2}{|l|}{ qPCR } & \multirow{3}{*}{$\begin{array}{l}\text { overall } \\
n=158\end{array}$} \\
\hline & & NPS & saliva & NPS & saliva & \\
\hline & & $\mathrm{n}=94$ & $\mathrm{n}=6$ & $n=121$ & $\mathrm{n}=93$ & \\
\hline \multicolumn{7}{|l|}{ study period } \\
\hline $\begin{array}{l}\text { Aug 2016-Jul } \\
2017\end{array}$ & 160 & $40(25 \%)$ & $6(4 \%)$ & 60 (38\%) & 46 (29\%) & 79 (49\%) \\
\hline $\begin{array}{l}\text { Aug 2017-Jul } \\
2018\end{array}$ & 106 & $23(22 \%)$ & 0 & $27(25 \%)$ & $19(18 \%)$ & $31(29 \%)$ \\
\hline $\begin{array}{l}\text { Aug 2018-Jul } \\
2019\end{array}$ & 97 & $24(25 \%)$ & 0 & 25 (26\%) & $23(28 \%)$ & 37 (38\%) \\
\hline $\begin{array}{l}\text { Aug 2019-Mar } \\
2020\end{array}$ & 31 & $7(23 \%)$ & 0 & 9 (29\%) & $5(16 \%)$ & 11 (35\%) \\
\hline \multicolumn{7}{|l|}{ seasons } \\
\hline spring-summer & 153 & $28(18 \%)$ & 1 (1\%) & 36 (24\%) & $25(16 \%)$ & $46(30 \%)$ \\
\hline \multirow[t]{2}{*}{ autumn-winter } & 241 & $66(27 \%) *$ & $5(2 \%)$ & $85(25 \%) * *$ & $68(28 \%) * *$ & $112(47 \%)$ \\
\hline & & $\begin{array}{l}R R \\
1.50(1.01- \\
2.22)\end{array}$ & $\begin{array}{l}R R \\
3.17(0.37- \\
26.9)\end{array}$ & $\begin{array}{l}R R \\
1.50(1.07- \\
2.09)\end{array}$ & $\begin{array}{l}R R \\
1.73(1.14- \\
2.61)\end{array}$ & $\begin{array}{l}R R 1.55 \\
(1.17-2.04)\end{array}$ \\
\hline \multicolumn{7}{|l|}{$\begin{array}{l}\text { child's age in } \\
\text { months }\end{array}$} \\
\hline $12-23$ & 147 & 42 (29\%) & $2(1 \%)$ & 52 (35\%) & $34(23 \%)$ & 62 (42\%) \\
\hline $24-35$ & 102 & 23 (23\%) & 0 & $28(27 \%)$ & $20(20 \%)$ & $36(35 \%)$ \\
\hline $36-47$ & 71 & $14(20 \%)$ & $2(3 \%)$ & $20(28 \%)$ & $19(27 \%)$ & $30(42 \%)$ \\
\hline $48-59$ & 74 & 15 (20\%) & $2(3 \%)$ & $21(28 \%)$ & $20(27 \%)$ & $30(41 \%)$ \\
\hline \multicolumn{7}{|l|}{ child's gender } \\
\hline female & 169 & $43(25 \%)$ & $2(1 \%)$ & $51(30 \%)$ & $41(24 \%)$ & 62 (37\%) \\
\hline \multirow[t]{2}{*}{ male } & 225 & 51 (23\%) & $4(2 \%)$ & 70 (31\%) & 52 (23\%) & 96 (43\%) \\
\hline & & $\begin{array}{l}R R \\
0.89(0.63- \\
1.27)\end{array}$ & $\begin{array}{l}R R \\
1.52(0.28- \\
8.11)\end{array}$ & $\begin{array}{l}R R \\
1.03(0.76- \\
1.39)\end{array}$ & $\begin{array}{l}R R \\
0.95(0.67- \\
1.36)\end{array}$ & $\begin{array}{l}R R \\
1.16(0.91- \\
1.49)\end{array}$ \\
\hline \multicolumn{7}{|l|}{$\begin{array}{l}\text { day -care } \\
\text { centre } \\
\text { attendance }\end{array}$} \\
\hline no & 187 & $28(15 \%)$ & 0 & $39(21 \%)$ & $22(12 \%)$ & $49(26 \%)$ \\
\hline
\end{tabular}

Fisher Exact, two-tailed, $p-*<0.05, * *<0.01, * * *<0.001$, and $* * * *<0.0001$, 


\begin{tabular}{|c|c|c|c|c|c|c|}
\hline \multirow[t]{4}{*}{ attribute } & \multirow{4}{*}{$\begin{array}{l}\text { number of } \\
\text { individuals }\end{array}$} & \multicolumn{5}{|c|}{ carriers detected with: } \\
\hline & & \multicolumn{2}{|c|}{ conventional culture } & \multicolumn{2}{|l|}{ qPCR } & \multirow{3}{*}{$\begin{array}{l}\text { overall } \\
n=158\end{array}$} \\
\hline & & NPS & saliva & NPS & saliva & \\
\hline & & $n=94$ & $n=6$ & $n=121$ & $n=93$ & \\
\hline \multirow[t]{2}{*}{ yes } & 207 & $\begin{array}{l}66(32 \%) \\
\star \star \star \star\end{array}$ & $6(3 \%) *$ & $\begin{array}{l}82(40 \%) \\
\star \star \star \star\end{array}$ & $\begin{array}{l}71(34 \%) \\
\star \star \star \star\end{array}$ & $109(53 \%)$ \\
\hline & & $\begin{array}{l}R R \\
2.13(1.43- \\
3.16)\end{array}$ & & $\begin{array}{l}R R \\
1.90(1.37- \\
2.63)\end{array}$ & $\begin{array}{l}R R \\
2.92(1.89- \\
4.51)\end{array}$ & $\begin{array}{l}R R \\
2.01(1.53- \\
2.64)\end{array}$ \\
\hline \multicolumn{7}{|c|}{$\begin{array}{l}\text { smoker in a } \\
\text { household }\end{array}$} \\
\hline no & 254 & $69(27 \%)$ & $6(2 \%)$ & 87 (34\%) & $66(26 \%)$ & 110 (43\%) \\
\hline \multirow[t]{2}{*}{ yes } & 139 & $25(18 \%) *$ & 0 & 34 (24\%) & 27 (19\%) & 48 (35\%) \\
\hline & & $\begin{array}{l}R R \\
0.89(0.80- \\
0.99)\end{array}$ & & $\begin{array}{l}R R \\
0.87(0.76- \\
0.99)\end{array}$ & $\begin{array}{l}R R \\
0.92(0.82- \\
1.02)\end{array}$ & $\begin{array}{l}R R \\
0.87(0.74- \\
1.02)\end{array}$ \\
\hline unknown & 1 & 0 & 0 & 0 & 0 & 0 \\
\hline \multicolumn{7}{|l|}{ siblings } \\
\hline no & 153 & $28(18 \%)$ & $2(1 \%)$ & 39 (25\%) & 37 (24\%) & $53(35 \%)$ \\
\hline \multirow[t]{2}{*}{ yes } & 240 & $66(28 \%) *$ & $4(2 \%)$ & $82(34 \%)$ & $56(23 \%)$ & 105 (44\%) \\
\hline & & $\begin{array}{l}R R \\
1.52(1.04- \\
2.89)\end{array}$ & & $\begin{array}{l}R R \\
1.34(0.97- \\
1.85)\end{array}$ & $\begin{array}{l}R R \\
0.96(0.67- \\
1.39)\end{array}$ & $\begin{array}{l}R R \\
1.26(0.97- \\
1.64)\end{array}$ \\
\hline unknown & 1 & 0 & 0 & 0 & 0 & 0 \\
\hline
\end{tabular}

\section{Carriage of S. pneumoniae}

Altogether, 158 (40.1\%) of 394 children have been identified as carriers of pneumococcus by any method used in the study. Ninety-seven children ( $25.4 \%$ of 394 ) have been identified as carriers of $S$. pneumoniae using conventional culture method (Fig. 1b). With 94 of these 97 children positive in NPS and six in saliva, NPS was far superior to saliva in the sensitivity of detecting pneumococcal carriage by isolation of live $S$. pneumoniae from a child (McNemar's, $p<$ $0.000001)$. All 394 saliva samples yielded a colony growth on GENT-agar and all these plates were harvested whereas $68(17.3 \%)$ of 394 NPS cultures were negative for any growth. We considered these 68 NPS to be negative for $S$. pneumoniae also by molecular method.

Samples from 155 children (39.1\% of 394) have been identified as positive for pneumococcus with qPCRs (Fig. 1b). It included all samples from which S. pneumoniae has been cultured except for three NPS samples positive by culture for non-typeable (NT) pneumococci (Fig. 1a), and a single saliva sample from which serotype 24F strain has been cultured (Fig. 1C). Similar to diagnostic culture, with molecular methods the number of positive results was higher for NPS compared to saliva (121 or 30.7\% versus 93 or $23.6 \%$; McNemar's, $p<0.01$ ) (Fig. 1b). However, unlike diagnostic 
culture, testing saliva on the top of NPS significantly increased the number S. pneumoniae carriers detected from 121 to 155 (Fisher Exact, $p<0.05$ ). Also, the number of carriers detected by testing the DNA extracted from culture-enriched saliva samples was not different compared with carriers detected by the gold standard of NPS culture (94 or $23.9 \%$ versus 93 or $23.6 \%$ of 394 ; McNemar's, $p=1.0$ ). Of note, 12 of 68 saliva samples from children with NPS cultures negative for any on-growth GENT-agar plates were positive for $S$. pneumoniae.

DCC attendance was associated with a higher risk ratio (RR) of pneumococcal carriage (RR2.13(1.43-3.16), $p<$ 0.0001 , and RR2.01(1.53-2.70), $p<0.0001$, when detected by culture of NPS and by any method, respectively, Table 1). RR was also elevated during autumn and winter months. Being a sibling was associated with a higher risk of carriage in the study (RR1.52(1.04-2.89), $p<0.05)$, but only when $S$. pneumoniae was detected by culture of NPS and was driven primarily by an effect observed in 12-23 month-olds (RR2.40(1.28-4.51), $p<0.001$ ) and not in other age groups (24-35 months old, $p=0.70 ; 36-47$ months old, $p=0.17 ; 48-59$ months old, $p=0.66$ ). After correcting for DCC attendance an impact of the siblings has become insignificant (RR1.02(0.76-1.38), $p=0.89)$. RR was higher in children from households without a smoker (Chi-square, $p<0.05$ ), but only when carriage was detected by culture of NPS. There was no effect of age or gender on prevalence of carriage detected by a particular method or overall (Table 1).

\section{Serotype carriage}

Serotypes of strains cultured from children and detected with qPCR in culture-enriched samples in the study are all listed in Table 2. Altogether 99 strains have been isolated from 97 children, as S. pneumoniae strains of two different serotypes have been cultured from NPS collected from two individuals. Ninety-three of these 99 isolates represented 26 different serotypes and the remaining six were classified as non-typeable pneumococci. The most common serotype among cultured S. pneumoniae strains were 23A and 6B isolated from 10 children each, followed by 15BC, $10 \mathrm{~A}$ and $11 \mathrm{~A}$ isolated from seven children each, and by serotypes $23 \mathrm{~B}$ and $35 \mathrm{~F}$ isolated from six children each. Strains of these seven serotypes constituted $53.5 \%$ of 99 strains cultured in the study. The coverage of PCV10 and PCV13 was $23.2 \%$ (23 of 99 ) and $26.3 \%$ (26 of 99 ), respectively. 
Table 2

Serotypes of strains cultured in the study or detected using serotype-specific and serogroup-specific qPCRs.

\begin{tabular}{|c|c|c|c|c|c|c|c|c|c|}
\hline \multirow[t]{2}{*}{ serotype } & \multicolumn{3}{|l|}{ NPS } & \multicolumn{3}{|l|}{ saliva } & \multicolumn{3}{|c|}{ NPS or saliva } \\
\hline & culture & qPCR & overall & culture & qPCR & overall & culture & qPCR & overall \\
\hline 1 & 0 & 1 & 1 & 0 & 0 & 0 & 0 & 1 & 1 \\
\hline 3 & 2 & 2 & 2 & 0 & 5 & 5 & 2 & 7 & 7 \\
\hline 4 & 0 & $\mathrm{NR}^{\#}$ & 0 & 0 & $\mathrm{NR}^{\#}$ & 0 & 0 & $\mathrm{NR}^{\#}$ & 0 \\
\hline 5 & 0 & $\mathrm{NR}^{\#}$ & 0 & 0 & $\mathrm{NR}^{\#}$ & 0 & 0 & $\mathrm{NR}^{\#}$ & 0 \\
\hline $\begin{array}{l}6 \\
(\mathrm{~A} / \mathrm{B} / \mathrm{C} / \mathrm{D})\end{array}$ & $\begin{array}{l}14 \\
(1 / 10 / 3 / 0)\end{array}$ & 15 & 16 & 0 & 15 & 15 & $\begin{array}{l}14 \\
(1 / 10 / 3 / 0)\end{array}$ & 22 & 23 \\
\hline 7 (A/F) & 0 & 0 & 0 & 0 & 0 & 0 & 0 & 0 & 0 \\
\hline 8 & 0 & 0 & 0 & 0 & 0 & 0 & 0 & 0 & 0 \\
\hline $\begin{array}{l}9 \\
(\mathrm{~A} / \mathrm{L} / \mathrm{N} / \mathrm{V})\end{array}$ & $\begin{array}{l}1 \\
(0 / 0 / 1 / 0)\end{array}$ & 9 & 9 & 0 & 10 & 10 & $\begin{array}{l}1 \\
(0 / 0 / 1 / 0)\end{array}$ & 17 & 17 \\
\hline 10 (A/B) & $7(7 / 0)$ & 6 & 7 & 0 & 2 & 2 & $7(7 / 0)$ & 7 & 8 \\
\hline 11 (A/D) & $7(7 / 0)$ & 10 & 10 & 0 & 8 & 8 & $7(7 / 0)$ & 14 & 14 \\
\hline $\begin{array}{l}12 \\
(\mathrm{~A} / \mathrm{B} / \mathrm{F})\end{array}$ & 0 & 1 & 1 & 0 & 4 & 4 & 0 & 4 & 4 \\
\hline 14 & 3 & 4 & 4 & 0 & 4 & 4 & 3 & 6 & 6 \\
\hline $\begin{array}{l}15 \\
(\mathrm{~A} / \mathrm{BC} / \mathrm{F})\end{array}$ & $13(6 / 7 / 0)$ & 22 & 22 & $\begin{array}{l}1 \\
(1 / 0 / 0)\end{array}$ & 12 & 12 & $13(6 / 7 / 0)$ & 24 & 24 \\
\hline $16 \mathrm{~F}$ & 0 & 0 & 0 & 0 & 0 & 0 & 0 & 0 & 0 \\
\hline $\begin{array}{l}18 \\
(\mathrm{~A} / \mathrm{B} / \mathrm{C} / \mathrm{F})\end{array}$ & $\begin{array}{l}1 \\
(0 / 0 / 1 / 0)\end{array}$ & 1 & 1 & 0 & 0 & 0 & $\begin{array}{l}1 \\
(0 / 0 / 1 / 0)\end{array}$ & 1 & 1 \\
\hline $19 A$ & 0 & 2 & 2 & 0 & 0 & 0 & 0 & 2 & 2 \\
\hline $19 \mathrm{~F}$ & 4 & 4 & 4 & 0 & 0 & 0 & 4 & 4 & 4 \\
\hline 20 & 0 & 0 & 0 & 0 & 0 & 0 & 0 & 0 & 0 \\
\hline 21 & 1 & 1 & 1 & 0 & 2 & 2 & 1 & 3 & 3 \\
\hline $22(\mathrm{~A} / \mathrm{F})$ & $3(0 / 3)$ & 8 & 8 & 0 & 3 & 3 & $3(0 / 3)$ & 10 & 10 \\
\hline $23 \mathrm{~A}$ & 10 & 11 & 12 & 0 & 10 & 10 & 10 & 17 & 18 \\
\hline 23B & 5 & 6 & 6 & 1 & 4 & 5 & 6 & 8 & 9 \\
\hline $23 F$ & 5 & 4 & 5 & 0 & 4 & 4 & 5 & 6 & 6 \\
\hline $\begin{array}{l}\text { \# - an assay } \\
\text { thus detect } \\
\text { cultured fro } \\
\text { pneumonia } \\
\text { of } S \text {. pneun }\end{array}$ & $\begin{array}{l}\text { f insufficien } \\
\text { by culture a } \\
97 \text { carriers; } \\
\text { oy molecular } \\
\text { niaeby any }\end{array}$ & $\begin{array}{l}\text { pecifici } \\
\text { ne; @ - r } \\
\text { numbe } \\
\text { nethod }\end{array}$ & $\begin{array}{l}y \text { thus co } \\
\text { on-typeal } \\
\text { of serot } \\
\text { qPCR); }\end{array}$ & $\begin{array}{l}\text { ed to be } \\
\text { oneumol } \\
\text { etected } \mathrm{i} \\
\text { er of sel }\end{array}$ & $\begin{array}{l}\text { t reliab } \\
\text { e strair } \\
55 \text { ind } \\
\text { ypes d }\end{array}$ & $\begin{array}{l}\text { (NR); \& } \\
\text { a - num } \\
\text { iduals id } \\
\text { ected in }\end{array}$ & $\begin{array}{l}\text { types not tars } \\
S \text {. pneumon } \\
\text { ed as carriers } \\
\text { idividuals ide }\end{array}$ & $\begin{array}{l}\text { ted by } F \\
\text { e strain } \\
\text { of } S \text {. } \\
\text { tified as }\end{array}$ & $\begin{array}{l}\text { IPCR, } \\
\text { carriers }\end{array}$ \\
\hline
\end{tabular}




\begin{tabular}{|c|c|c|c|c|c|c|c|c|c|}
\hline \multirow[t]{2}{*}{ serotype } & \multicolumn{3}{|l|}{ NPS } & \multicolumn{3}{|l|}{ saliva } & \multicolumn{3}{|c|}{ NPS or saliva } \\
\hline & culture & qPCR & overall & culture & qPCR & overall & culture & qPCR & overall \\
\hline $24 F^{8}$ & 2 & - & 2 & 2 & - & 2 & 2 & - & 2 \\
\hline $28 \mathrm{~F}^{8}$ & 2 & - & 2 & 0 & - & 0 & 2 & - & 2 \\
\hline $31^{\&}$ & 1 & - & 1 & 1 & - & 1 & 2 & - & 2 \\
\hline 33 (A/F) & 0 & 1 & 1 & 0 & 3 & 3 & 0 & 3 & 3 \\
\hline 34 & 0 & 0 & 0 & 0 & 0 & 0 & 0 & 0 & 0 \\
\hline $35 A^{\&}$ & 1 & - & 1 & 0 & - & 0 & 1 & - & 1 \\
\hline $35 \mathrm{~B}$ & 1 & 2 & 2 & 0 & 6 & 6 & 1 & 7 & 7 \\
\hline $35 F^{\&}$ & 6 & - & 6 & 0 & - & 0 & 6 & - & 6 \\
\hline $37^{\&}$ & 1 & - & 1 & 0 & - & 0 & 1 & - & 1 \\
\hline 38 & 1 & 3 & 3 & 0 & 3 & 3 & 1 & 5 & 5 \\
\hline NT@ & 5 & - & 5 & 1 & - & 1 & 6 & - & 6 \\
\hline Total & 96 & 112 & 135 & 6 & 95 & 32 & $99^{a}$ & $168^{b}$ & $192^{c}$ \\
\hline \multicolumn{10}{|c|}{$\begin{array}{l}\text { \# - an assay of insufficient specificity thus considered to be not reliable (NR); }{ }^{\&} \text { - serotypes not targeted by RT-PCR, } \\
\text { thus detected by culture alone; } @ \text { - non-typeable } S \text {. pneumoniae strains; }{ }^{a} \text { - number of } S \text {. pneumoniae strains } \\
\text { cultured from } 97 \text { carriers; }{ }^{b} \text { - number of serotypes detected in } 155 \text { individuals identified as carriers of } S \text {. } \\
\text { pneumoniae by molecular method (qPCR); }{ }^{c} \text { - number of serotypes detected in } 158 \text { individuals identified as carriers } \\
\text { of } S \text {. pneumoniae by any method used in the study. }\end{array}$} \\
\hline
\end{tabular}

The qPCR assays applied to detect serotype-specific sequences in DNA extracted from culture-enriched samples covered serotypes of 79 (84.9\%) of 93 encapsulated S. pneumoniae strains cultured. Remaining 14 encapsulated strains were of serotype $35 \mathrm{~F}(n=6), 24 \mathrm{~F}$ and $28 \mathrm{~F}(\mathrm{n}=2$ each), and $31,35 \mathrm{~A}$, and 37 (single isolate each) (Table 2). Application of qPCR lead to the detection of 168 serotype-carriage events in $124(78.5 \%)$ of 158 of children classified as carriers by any method used. Of these 168, 112 events have been detected in 93 NPS and 95 in 68 saliva samples, representing $75.0 \%$ of all 124 NPS samples and $72.3 \%$ of all 94 saliva samples positive by any method for $S$. pneumoniae. The remaining 57 samples $(26.1 \%)$ with no signal for any serotype were 28 NPS and 25 saliva collected from 33 carriers. In line with previous reports [26, 28], qPCRs targeting serotypes 4 and 5 showed a lack of specificity when applied to both, NPS and to saliva samples. Results of these two assays were excluded from analysis.

Among 158 children classified as carriers of $S$. pneumoniae by any method, the most common serotype/serogroup detected with qPCRs were: serogroup 15 ( $n=24$ or $15.2 \%$ of 158 children), serogroup 6 ( $n=22$ or $13.9 \%$ ), serogroup 9 and serotype $23 \mathrm{~A}$ ( $\mathrm{n}=17$ or $10.8 \%$, each). For NPS, numbers of samples positive for serotype or serogroup by culture correlated strongly with the number of samples positive for the same serotypes by qPCR (Spearman's rho $=0.855, p<$ 0.0001) (Fig. 2a). There was also correlation between the number of serotype carriers detected with qPCR in NPS and in saliva (rho $=0.667, p<0.005$ ) (Fig. $2 \mathrm{~b}$ ), as well as between numbers of serotype-carriers detected with qPCR in NPS or in saliva versus overall cultured in the study (rho $=0.771, p<0.0001$ ) (Fig. 2 c). 
Although the number of VT strains cultured from children attending DCC ( $n=17$ of PCV10-VT and $n=20$ of PCV13-VT) was significantly higher compared with the number of cultured children staying home ( $\mathrm{n}=6$ of PCV10-VT and PCV13VT, Chi-square, $p=0.034$ and $p=0.01$, for difference in prevalence of PCV10-VTs and PCV13-VTs, respectively), there were no differences in fractions of PCV10-VT strains among all cultured from DCC attendees compared with children staying home (17 of 71 versus 6 of 28, Chi-square $p=0.79$ ), nor in fractions of PCV13-VT strains (20 of 71 for PCV10 versus 6 of 28 , Chi-square $p=0.49$ ). None of other demographic or environmental factors were associated with differences in serotype carriage.

\section{Co-carriage of multiple serotypes}

Presence of two or more serotypes was detected in 40 (25.3\%) of 158 children identified as carriers by either culture or using piaB and lytA qPCRs (Table 3). Co-carriage of two or more serotypes/serogroups was detected in $17.7 \%$ (22 of 124) NPS and $25.5 \%$ (20 of 94) saliva samples classified as positive for $S$. pneumoniae by any method. There was no significant difference in prevalence of co-carriage in NPS and saliva samples (Chi-square, $p=0.43$ ). Also, there were no statistically significant differences in co-carriage according to age (12-23 months old, 14 of 48; 24-35 months old, 5 of 31; 36-47 months old 10 of 25; 48-59 months old, 6 of 20; Chi-square $p>0.05$ between any two age groups), DCC attendance (yes 28 of 88 versus no 7 of 36, Chi-square, $p=0.17$ ), having siblings (yes 19 of 79 versus no 16 of 45 , Chisquare, $p=0.17$ ) nor the presence of a smoker in the child's household (yes 13 of 35 versus no 22 of 89 , Chi-square, $p$ $=0.17)$.

Table 3

Number of serotypes/serogroups detected with any method

\begin{tabular}{|llll|}
\hline number of detected serotypes & NPS & saliva & overall \\
\hline $\mathbf{0}$ & 270 & 300 & 236 \\
\hline $\mathbf{1}^{\#}$ & 102 & 74 & 118 \\
\hline $\mathbf{2}$ & 19 & 13 & 29 \\
\hline $\mathbf{3}$ & 3 & 5 & 8 \\
\hline $\mathbf{4}$ & 0 & 2 & 3 \\
\hline$\#_{\text {- includes samples pneumococcus-positive in qPCR with no signal for any serotype }}$ \\
\hline
\end{tabular}

\section{Temporal changes in serotype carriage}

To assess the presence of indirect effects of PCV implementation into NIP in January 2017 on serotype carriage in unvaccinated children, we compared timing of isolation of $S$. pneumoniae strains of serotypes targeted by PCV10 (PCV10-VTs) versus non-PCV10, and also timing of isolation PCV13-VTs versus non-PCV13 from carriers. We assumed that indirect effects of PCV implementation will drive the median time of a VT carriage detection towards an earlier time-point. There were no significant differences in fraction of PCV10-VTs and PCV13-VTs before (8 and 9 of 50, respectively) and after (15 and 17 of 50, respectively) the 50th of 99 strains has been cultured in the study in October 2017. Instead, there was a trend towards an increase in VTs fractions over time (Fisher Exact, $p=0.15$ and $p=0.11$ for PCV10-VTs and PCV13-VTs, respectively).

\section{Discussion}

There is little data published on pneumococcal carriage in Poland. Over the past two decades reports of only two Polish studies investigating the carriage of $S$. pneumoniae have been published. The first study was conducted 
between November 2000 and May 2001 in the capital city of Warsaw [14, 15]. The second study took place between November 2002 and June 2003 in Lublin [16, 17]. Both these studies have been conducted before the first of the PCVs, the heptavalent vaccine, became available commercially in the country. In light of obligatory pneumococcal vaccination for children born after 31 st of December 2016, data on pneumococcal carriage is needed. Here, we investigated S. pneumoniae carriage in unvaccinated toddlers in the country with PCV10 and PCV13 commercially available for over five years and at the time of PCV10 implementation into infants' immunization program. Our first goal was to establish a baseline for future studies investigating an impact of PCV10 introduction into NIP on S. pneumoniae carriage. The second goal was to assess suitability of testing oral fluids for pneumococcal carriage detection by comparing saliva testing with the gold standard method of conventional diagnostic culture of NPS.

Conventional diagnostic culture was the only method used to detect $S$. pneumoniae in previous studies conducted in Poland between 2000 and 2003 [14-17]. The carriage rate of $25.4 \%$ we detected with that method in our cohort was lower compared with $45.7-54.6 \%$ reported by Sulikowska et al. [14, 15] and 33.1-44.4\% by Korona-Glowniak et al. [16, 17]. However, the differences in cohorts' demographic composition, methods of sampling, and seasons of samples collection, make interpretation of differences in S. pneumoniae carriage difficult.

It is documented that DCC attendance increases pneumococcal transmission [29-31]. Also in all three Polish studies, our study included, the risk of pneumococcal carriage was significantly higher in children attending DCCs compared to staying home. Carriage rate of $31.9 \%$ detected by us with conventional culture of NPS in children attending DCCs was significantly different from $54.2-56.5 \%$ by Sulikowska et al. $[14,15]$ (Chi-square, $p<0.005)$. Hence, the higher proportion of children staying home in our study (47.5\%) compared with cohort investigated by Sulikowska et al. [14, 15] $(25.7 \%)$ and by Korona-Glowniak et al. [16, 17] $(22.3 \%)$, per se contributed to lower rate of S. pneumoniae carriage. Interestingly, there was no significant difference between rate of $15.0 \%$ we detected in home-setting children compared with $19.3-25.9 \%$ reported by Sulikowska et al. (Chi-square, $p>0.1$ ). Also, children have been enrolled into our study all year long whereas in the previous two exclusively during autumn and winter, the seasons when carriage rates are higher [31, this study]. It seems very likely that the continuous enrolment contributed further to lower carriage rates in our study. Finally, higher $S$. pneumoniae carriage reported by Korona-Glowniak et al. [16, 17] can be attributed to oropharyngeal samples (OPS) being cultured on the top of NPS in their study. When excluding results for OPS, there was no difference in point-prevalence of $25.4 \%$ detected with NPS culture by us and $24.4 \%$ detected by KoronaGlowniak et al. [16, 17] ( $\mathrm{n}=94$ of 394 versus $\mathrm{n}=228$ of 933 , Chi-square, $p=0.88)$. In conclusion, there is no evidence for any substantial indirect effects of commercial use of PCVs on rates of overall pneumococcal carriage in unvaccinated children (detected by culture).

There was, however, a reduction in proportions of PCV10-VTs (23.2\% versus $73.4 \%)$ and PCV13-VTs (26.3\% versus $80.4 \%$ ) among the strains cultured in our study, when compared to the 2002-2003 study conducted by KoronaGlowniak et al. [16, 17] prior to PCVs entering the Polish market. This reduction in VTs carriage in unvaccinated children represents a heard effect of commercial vaccination with PCVs prior to and independent from PCV10 implementation into the NIP. Also, instead of a decline of PCV10-VTs carriage over the study period there was, albeit not statistically significant, an increase in a fraction of VTs among all strains cultured. It also points at commercial vaccination prior to PCV implementation into NIP as the dominant force behind the herd effect. It also suggests to us that study results can be considered to represent the baseline for future surveillance of impact of PCVs introduction into NIP on carriage.

One noticeable result was low prevalence of serotype 19A in carriage. Serotype 19A has been reported to emerge at various sites in replacement after PCV7 implementation [7, 32] and also to persist in carriage in populations with infants vaccinated with PCV10 $[33,34]$. Here, carriage of $19 \mathrm{~A}$ was detected in only two children and exclusively with

Page $11 / 19$ 
the molecular method. With estimated $25-30 \%$ of infants being vaccinated in Poland with PCV13 outside NIP, low presence of this PCV13-VT could reflect indirect PCV13 effects. It also indicates a high IPD cases to carriers rate (CCR), thus high invasiveness of serotype 19A strains in Poland, because between 2016 and 2020 (time of present study) serotype 19A was, after serotype 14, the second most common in IPD in Polish children aged 12-59 months, whereas in our study serotype $19 \mathrm{~A}$ ranked 19 th in frequency in carriage [6].

Importantly, low numbers of VTs cultured from unvaccinated children make future assessment of direct effects of PCV implementation into NIP on VTs carriage difficult. The solution could be increasing the power of the next study by sampling higher numbers of subjects and/or detecting carriage of serotypes with a substantially more sensitive diagnostic approach. The latter can be addressed by testing multiple samples per child [16, 17] and/or employing molecular detection methods.

In our study, application of molecular diagnostics and testing saliva on the top of NPS increased the number of carriers detected by a factor of 1.7 , from ninety-four identified by gold standard of conventional culture of NPS to an overall number of 158. In both materials, NPS and saliva, the application of molecular methods significantly increased the sensitivity of $S$. pneumoniae detection. This is in line with results reported by Wyllie et al. in studies applying a similar protocol to test NPS from children and to test NPS and saliva samples from adults, conducted between 2014 and 2016 in the Netherlands [20,33].

NPS is the specimen recommended by WHO in pneumococcal carriage detection in children [19] and it has been reported that NPS is a more valuable material than OPS [35]. In our study, the culture of NPS was far superior to culture of saliva and also, $39.6 \%$ of carriers (61 of 154 ) identified by qPCR were detected by NPS only. However, with $21.5 \%$ (34 of 158) of carriers detected exclusively in saliva, testing oral fluids substantially increased the number of carriers detected. In line with this finding, Korona-Glowniak et al. $[16,17]$ also reported that testing OPS along NPS significantly increased the number of carriers detected, and that there was no difference between the number of carriers detected by culturing NPS compared with OPS $[16,17]$. Therefore, the best carriage detection might be achieved by testing from each individual multiple specimens, e.g. NPS, OPS and saliva, or a combination of any two of these.

Molecular methods appeared to be superior to conventional culture in detecting co-carriage of multiple serotypes in this study (2.1\% in culture versus $26.6 \%$ in qPCR). Wyllie et al. [33] and Kandasamy et al. [36] obtained similar levels of multiple serotype carriage using molecular methods. The higher sensitivity of any minority serotype detection in multiserotype carriage allowed for a more detailed analysis of the occurrence of serotypes. Since available qPCR assays did not cover all serotypes, and not always distinguished serotypes within a serogroup, the number of multi-serotype carriers still might be understated.

Among our study limitations was a lack of molecular assays that would detect carriage of every circulating serotype. For example, strains of serotypes 24F, 28F, 35A, 35F, and 38 have been cultured from children, yet none of these serotypes were targeted with qPCR. Another limitation was low resolution of certain qPCRs not discriminating between serotypes within a serogroup, with 10 out of 27 qPCR assays targeting more than one serotype. A limitation was also the low sensitivity of conventional diagnostic culture. It concerns both sample types, but due to very rich bacterial growth, including many non-pneumococcal a-hemolytic colonies, it was particularly difficult to isolate live $S$. pneumoniae from saliva. With large numbers of serotype-carriage events detected exclusively with qPCR, and in the light of reports on non-pneumococcal streptococci expressing the pneumococcal capsular polysaccharides [28, 37], we paid particular attention to the specificity of assays. We addressed it by testing for serotype samples negative for $S$. pneumoniae and excluding the results of assays that generated a positive result (serotype 4 and serotype 5 specific qPCRs). Nevertheless, we can't exclude that some of the results represent carriage of confounded non-pneumococcal bacteria detected with qPCRs. For example, when applied to oropharyngeal and saliva samples from adults, a 
diminished specificity of serogroup 9-specific assay has been reported $[26,28]$ and serogroup 9 was the clear outlier when culture data was compared with qPCR results in our study (Table 2 and Fig. 2a and 2c). However, since we did not observe positivity in this assay among samples negative for $S$. pneumoniae, nor was there a difference between the number of NPS and saliva samples positive for this serogroup by qPCR, and we are not aware of any reports on the assay's poor specificity in NPS from children, we consider results for serogroup 9 as reliable.

In summary, pneumococcal carriage rate detected with WHO's recommended method in Polish children was lower compared with studies conducted prior to the introduction of commercial PCVs in the country, yet we attribute it to differences in set-ups of studies rather than the effect of PCVs. On the other hand, the decline in prevalence of PCV10VTs and PCV13-VTs carriage compared with the pre-PCV period suggests strong herd effects of commercial vaccination independent of NIP in Poland. According to the results obtained in our study, NPS was a more valuable material in carriage detection in children and qPCR was the more sensitive method in pneumococcal and pneumococcal serotype carriage detection. Also, information about carriage rate and serotype distribution among unvaccinated Polish children gained during this study can be used as a baseline in future carriage projects. The knowledge concerning the methods used in pneumococcal carriage detection gained during our study can be used for further research.

\section{Materials And Methods}

\section{Study design}

The study was performed between August 2016 and March 2020 among children age 12 to 59 months not vaccinated with any pneumococcal vaccine and attending a 'non-sick-visit' in hospital outpatients' clinics or community healthcare centers in cities of Warsaw and Wroclaw. First, parents (or child's legal guardians) were asked if family would be interested in participation in the study. If they responded positively, they were informed about the study goals and procedures and asked to give written informed consent for the child's participation. Next, parents were asked to fill-in the questionnaire and provide information on the child's age, gender, environment (number of siblings, day-care attendance, presence of smoker in child's household), and clinical information (pneumococcal vaccination, reason of doctor's office visit, occurrence of chronic diseases, symptoms of lower respiratory tract infections, antibiotic therapy in the past three months). The questionnaire was reviewed on site by the study personnel to exclude children that have been vaccinated with any pneumococcal vaccine, were treated within last 4 weeks with any antibiotic, have any immunodeficiency or symptom of lower respiratory tract infections. Finally, a saliva sample and nasopharyngeal swab were collected from each child by the study medical personnel.

\section{Ethical statement}

The study was approved by Bioethics Committee of Military Institute of Medicine in Warsaw (Resolution No. 49/WIM/2016) and was conducted in accordance with the World Health Medical Association 1966 Declaration of Helsinki and the EU rules of Good Clinical Practice.

\section{Collection of samples}

Saliva samples were collected using Oracol+® kit (Malvern Medical Developments Ltd., UK) as previously described [25]. NPS were collected using ESwab ${ }^{\text {TM }} 482 \mathrm{C}$ (COPAN Diagnostics, Brescia, Italy) and immediately placed in $1 \mathrm{ml}$ Amies transport medium (COPAN Diagnostics). Within 32 hours samples were delivered to the NRCBM and either immediately cultured or supplemented on arrival with $10 \%$ glycerol and stored at $-70^{\circ} \mathrm{C}$. 
NPS samples and extracted from Oracol+ $₫$ saliva samples were plated on Columbia Agar (Becton Dickinson, USA) $7 \%$ Sheep blood (Graso, Poland) with $5 \mu \mathrm{g} / \mathrm{ml}$ gentamycin (Sigma-Aldrich, USA) (GENT-agar) and incubated for 18-24h in $35^{\circ} \mathrm{C}, 5 \% \mathrm{CO}_{2}$ as previously described $[38,20]$. Once pneumococcus-like colonies were re-plated, all remaining colony growth was harvested [20]. These harvests represented samples culture-enriched (CE) for S. pneumoniae [38]. Replated isolates were identified as $S$. pneumoniae based on susceptibility to optochin (BioMerièux, France) and bile solubility (Becton Dickinson, USA) [39].

Molecular detection of S. pneumoniae

The DNA was extracted from $200 \mu$ of culture-enriched NPS and culture-enriched saliva samples using SaMag- $12^{\text {TM }}$ System (Sacace Biotechnologies, Italy). The DNA was eluted into $100 \mu \mathrm{l}$ sample volume and stored in $2-8^{\circ} \mathrm{C}$. Pneumococcal DNA was detected with qPCR by testing $0.625 \mu \mathrm{l}$ of eluted sample in $25 \mu \mathrm{l}$ reaction volume, using TaqMan Universal MasterMix (Life Technologies, USA), and primers and probes targeting sequences within piaB and lytA genes $[38,40]$. A sample was considered positive for pneumococcus when both signals were $<40 \mathrm{C}_{T}$.

\section{Serotyping of S. pneumoniae strains}

Cultured pneumococci were serotyped using ImmuLex ${ }^{\text {TM }}$ Pneumotest Kit (SSI Diagnostica, Denmark), PCR, gene sequencing or Quellung reaction as previously described [41-44].

\section{Molecular detection of S. pneumoniae serotypes}

DNA extracted from culture-enriched NPS and saliva was tested with qPCR for presence of serotype-specific sequences as described before [28] using primers and probes for serotype 1, 3, 4, 5, 6A/B/C/D, 7A/F, 8, 9A/L/N/V, 10A/B, 12A/B/F, 14, 15A/BC/F, 19A, 20, 21, 22AF, 23A, 23B, 23F, 33A/F, 35B/C, and 38 published by Azzari et al. [45], for serotype 16F published by Azzari et al. [32], for serotypes 11A/D, 18A/B/C/F, and 19F published by Pimenta et al. [46], and for serotype 34 published by Sakai et al. [47]. A sample was considered positive for pneumococcus when the signal detected in serotype-specific qPCR was $<40 \mathrm{C}_{T}$. All culture-enriched samples classified with molecular methods as positive and two hundred randomly selected culture-enriched samples classified as negative for S. pneumoniae (100 NPS and saliva samples each) have been tested with qPCR for serotypes detection. Results of assays generating positive signal in samples classified as negative for pneumococcus were interpreted as unreliable and excluded from analysis.

\section{Statistics}

The Chi-square, Fisher Exact, and McNemar's tests were used for determination of statistical significance $(p<0.05)$. Data on serotype prevalence detected using different methods were correlated using Spearman's correlation test for nonparametric data.

\section{Declarations}

Acknowledgements: This work was supported by Pfizer via unrestricted grant (Investigator Initiated Research project WI218378) to the National Medicines Institute and to A.S. (the principal investigator). We are grateful to the participating families for commitment to the study. We wish to thank Prof. Waleria Hryniewicz and Prof. Ron Dagan for their advice on the study design. 
Author Contributions: A.S. and K.T. conceived the idea for the study. K.K., A.S. and K.T. wrote the study protocols. A.S. managed the study. M.W-R., D.R., A.G-K., K.W., A.T-C., T.J., J.C., W.M., A.M-M. and GIL Study Team recruited children to the study and collected material and data. I.W-P., A.S. and K.T. contributed analytical tools. I.W-P. and P.R. performed the assays. I.W-P., A.S. and K.T. analyzed and interpreted the data. I.W-P. and K.T. visualized the presentation of the results. I.W-P. drafted the manuscript. All authors reviewed, critically revised and approved the manuscript.

\section{Competing financial interests:}

M.W-R. has received speaking fees from Pfizer and GlaxoSmithKline. A.G-K. has received speaker fees from Pfizer. T.J. has received grants and non-financial support from Pfizer, and personal fees (for being on an advisory board and speaker fees) from Pfizer, Merck Sharp \& Dohme, and Sanofi Pasteur. A.M-M. has received fees for participation in advisory boards and speaking fees from Pfizer. A.S. has received grants and non-financial support from Pfizer, and personal fees (for being on an advisory board and speaker fees) from Pfizer, Merck Sharp \& Dohme and Sanofi Pasteur. K.T. has received consultation fees, fees for participation in advisory boards, speaking fees and funds for unrestricted research grants from Pfizer, funds for an unrestricted research grant from GlaxoSmithKline, and fees for participating in advisory boards from Merck Sharp \& Dohme, all paid directly to his home institution. L.S. has received personal fees (for being on an advisory board and speaker fees) from Pfizer, GlaxoSmithKline, Merck Sharp \& Dohme and Sanofi Pasteur. All other authors declare no competing interests.

\section{References}

1. CDC. Invasive pneumococcal disease - Annual Epidemiological Report for 2018. https://www.ecdc.europa.eu/en/publications-data/invasive-pneumococcal-disease-annual-epidemiological-report2018 (2020).

2. WHO. Pneumococcal vaccines WHO position paper. WER, 94, 85-104 (2019).

3. AlonsoDeVelasco, E., Verheul, A. F., Verhoef, J. \& Snippe, H. Streptococcus pneumoniae: virulence factors, pathogenesis, and vaccines. Microbiol. Rev, 59, 591-603 (1995).

4. Geno, K. A. et al. Pneumococcal capsules and their types: past, present, and future. Clin. Microbiol. Rev, 28, 871899 (2015).

5. Golicki, D., Dobrowolska, I. \& Gawrońska, A. Reprint: Rozwój i stan obecny szczepień przeciwko pneumokokom w Polsce.Standardy Medyczne Pediatria2 (2020).

6. Skoczyńska, A. et al. Inwazyjna choroba pneumokokowa w Polsce.NRCBM datahttps://koroun.nil.gov.pl/daneepidemiologiczne/ (2016- 2020).

7. Polkowska, A., Rinta-Kokko, H., Toropainen, M., Palmu, A. A. \& Nuorti, J. P. Long-term population effects of infant 10-valent pneumococcal conjugate vaccination on pneumococcal meningitis in Finland. Vaccine, 29, https://doi.org/10.1016/j.vaccine.2021.02.030 (2021).

8. Gladstone, R. A. et al. Five winters of pneumococcal serotype replacement in UK carriage following PCV introduction. Vaccine, 33, 2015-2021 (2015).

9. Richter, L. et al. Invasive pneumococcal diseases in children and adults before and after introduction of the 10valent pneumococcal conjugate vaccine into the Austrian national immunization program. PLoS One, 14, e0210081 https://doi.org/10.1371/journal.pone.0210081 (2019).

10. Fleming-Dutra, K. E. et al. Systematic review of the effect of pneumococcal conjugate vaccine dosing schedules on vaccine-type nasopharyngeal carriage. Pediatr. Infect. Dis. J, 33 (Suppl. 2), S152-S160 (2014). 
11. Davis, S. M., Deloria-Knoll, M., Kassa, H. T. \& O'Brien, K. L. Impact of pneumococcal conjugate vaccines on nasopharyngeal carriage and invasive disease among unvaccinated people: review of evidence on indirect effects. Vaccine, 32, 133-145 (2013).

12. Henriques-Normark, B. \& Tuomanen, E. I. The pneumococcus: epidemiology, microbiology, and pathogenesis. Cold Spring Harb. Perspect. Med, 3, a010215 https://doi.org/10.1101/cshperspect.a010215 (2013).

13. Auranen, K. et al. Colonisation endpoints in Streptococcus pneumoniae vaccine trials. Vaccine, 32, 153-158 (2013).

14. Sulikowska, A., Grzesiowski, P., Taraszkiewicz, M. \& Hryniewicz, W. The carriage of Streptococcus pneumoniae in the nasopharynx of children under 5 years of age in selected settings in Warsaw. Pediatr. Pol, 5, 377-384 (2003).

15. Sulikowska, A., Grzesiowski, P., Sadowy, E., Fiett, J. \& Hryniewicz, W. Characteristics of Streptococcus pneumoniae, Haemophilus influenzae, and Moraxella catarrhalis isolated from the nasopharynges of asymptomatic children and molecular analysis of S. pneumoniae and $\mathrm{H}$. influenzae strain replacement in the nasopharynx. J. Clin. Microbiol. 42, 3942-3949(2004).

16. Korona-Glowniak, I., Niedzielski, A. \& Malm, A. Upper respiratory colonization by Streptococcus pneumoniae in healthy pre-school children in south-east Poland. Int. J. Pediatr. Otorhinolaryngol, 75, 1529-1534 (2011).

17. Korona-Glowniak, I. \& Malm, A. Characteristics of Streptococcus pneumoniae strains colonizing upper respiratory tract of healthy preschool children in Poland. ScientificWorldJournal 2012, 732901 (2012).

18. O'Brien, K. L., Nohynek, H. \& World Health Organization Pneumococcal Vaccine Trials Carriage Working Group. Report from a WHO Working Group: standard method for detecting upper respiratory carriage of Streptococcus pneumoniae. Pediatr. Infect. Dis. J, 22, e1-11 https://doi.org/10.1097/01.inf.0000049347.42983.77 (2003).

19. Satzke, C. et al. Standard method for detecting upper respiratory carriage of Streptococcus pneumoniae: updated recommendations from the World Health Organization Pneumococcal Carriage Working Group. Vaccine, 32, 165179 (2013).

20. Wyllie, A. L. et al. Streptococcus pneumoniae in saliva of Dutch primary school children. PLoS One, 9, e102045 https://doi.org/10.1371/journal.pone.0102045 (2014).

21. Arguedas, A. et al. Upper respiratory tract colonization with Streptococcus pneumoniae in adults. Expert Review of Vaccines, 19, 353-366 (2020).

22. Almeida, S. T., Pedro, T., Paulo, A. C., de Lencastre, H. \& Sá-Leão, R. Re-evaluation of Streptococcus pneumoniae carriage in Portuguese elderly by qPCR increases carriage estimates and unveils an expanded pool of serotypes. Sci. Rep, 10, 8373 https://doi.org/10.1038/s41598-020-65399-x (2020).

23. Satzke, C. et al. The PneuCarriage Project: A multi-centre comparative study to identify the best serotyping methods for examining pneumococcal carriage in vaccine evaluation studies. PLoS Med, 12, e1001903 https://doi.org/10.1371/journal.pmed.1001903 (2015).

24. Olwagen, C. P., Adrian, P. V. \& Madhi, S. A. Comparison of traditional culture and molecular qPCR for detection of simultaneous carriage of multiple pneumococcal serotypes in African children. Sci. Rep, 7, 4628 https://doi.org/10.1038/s41598-017-04915-y (2017).

25. Krone, C. L. et al. Carriage of Streptococcus pneumoniae in aged adults with influenza-like-illness. PLoS One, 10, e0119875 https://doi.org/10.1371/journal.pone.0119875 (2015).

26. Wyllie, A. L. et al. Molecular surveillance on Streptococcus pneumoniae carriage in non-elderly adults; little evidence for pneumococcal circulation independent from the reservoir in children. Sci. Rep, 6, 34888 https://doi.org/10.1038/srep34888 (2016). 
27. Miellet, W. R. et al. Influenza-like illness exacerbates pneumococcal carriage in older adults. Clin. Infect. Dis. Preprint at https://academic.oup.com/cid/advance-article/doi/10.1093/cid/ciaa1551/5943453 (2020).

28. Wyllie, A. L. et al. Sequencing of the variable region of rpsB to discriminate between Streptococcus pneumoniae and other streptococcal species. Open Biol, 7, 170074 https://doi.org/10.1098/rsob.170074 (2017).

29. Principi, N., Marchisio, P., Schito, G. C. \& Mannelli, S. Risk factors for carriage of respiratory pathogens in the nasopharynx of healthy children. Ascanius Project Collaborative Group. Pediatr. Infect. Dis. J, 18, 517-523 (1999).

30. Pebody, R. G. et al. Use of antibiotics and risk factors for carriage of Streptococcus pneumoniae: a longitudinal household study in the United Kingdom. Epidemiol. Infect, 137, 555-561 (2009).

31. Ueno, M. et al. Prevalence and risk factors of nasopharyngeal carriage of Streptococcus pneumoniae in healthy children in Japan. Jpn. J. Infect. Dis, 66, 22-25 (2013).

32. Azzari, C. et al. Potential serotype coverage of three pneumococcal conjugate vaccines against invasive pneumococcal infection in Italian children. Vaccine, 30, 2701-2705 (2012).

33. Wyllie, A. L. et al. Molecular surveillance of nasopharyngeal carriage of Streptococcus pneumoniae in children vaccinated with conjugated polysaccharide pneumococcal vaccines. Sci. Rep, 6, 23809 https://doi.org/10.1038/srep23809 (2016b).

34. Tin, T. et al. The impact of pneumococcal conjugate vaccines on serotype 19A nasopharyngeal carriage. Expert Rev Vaccines, 18, 1243-1270 (2019).

35. Greenberg, D. et al. Relative importance of nasopharyngeal versus oropharyngeal sampling for isolation of Streptococcus pneumoniae and Haemophilus influenzae from healthy and sick individuals varies with age. J. Clin. Microbiol, 42, 4604-4609 (2004).

36. Kandasamy, R. et al. Multi-serotype pneumococcal nasopharyngeal carriage prevalence in vaccine naïve Nepalese children, assessed using molecular serotyping. PLoS One, 10, e0114286

https://doi.org/10.1371/journal.pone.0114286 (2015).

37. Gertz, R. E. Jr. Et al. Nonpneumococcal strains recently recovered from carriage specimens and expressing capsular serotypes highly related or identical to pneumococcal serotypes $2,4,9 \mathrm{~A}, 13$, and 23A. mBio, 12, e0103721 https://doi.org/10.1128/mBio.01037-21 (2021).

38. Trzciński, K. et al. Superiority of trans-oral over trans-nasal sampling in detecting Streptococcus pneumoniae colonization in adults. PLoS One, 8, e60520 https://doi.org/10.1371/journal.pone.0060520 (2013).

39. CDC. Chapter 8: Identification and characterization of Streptococcus pneumoniae in Laboratory methods for the diagnosis of meningitis caused by Neisseria meningitidis, Streptococcus pneumoniae, and Haemophilus influenzae (Second Edition, 2011).

40. Carvalho, M. D. G. et al. Evaluation and improvement of real-time PCR assays targeting lytA, ply, and psaA genes for detection of pneumococcal DNA. J. Clin. Microbiol, 45, 2460-2466 (2007).

41. Lawrence, E. R., Griffiths, D. B., Martin, S. A., George, R. C. \& Hall, L. M. C. Evaluation of semiautomated multiplex PCR assay for determination of Streptococcus pneumoniae serotypes and serogroups. J. Clin. Microbiol, 41, 601607 (2003).

42. Pai, R., Limor, J. \& Beall, B. Use of pyrosequencing to differentiate Streptococcus pneumoniae serotypes $6 \mathrm{~A}$ and 6B. J. Clin. Microbiol, 43, 4820-4822 (2005).

43. Pai, R., Gertz, R. E. \& Beall, B. Sequential multiplex PCR approach for determining capsular serotypes of Streptococcus pneumoniae isolates. J. Clin. Microbiol, 44, 124-131 (2006).

44. Neufeld, F. Über die agglutination der pneumokokken und über die theorie der agglutination. zeitschrift für hygiene und infektionskrankheiten, 40, 54-72 (1902). 
45. Azzari, C. et al. Realtime PCR is more sensitive than multiplex PCR for diagnosis and serotyping in children with culture negative pneumococcal invasive disease. PLoS One, 5, e9282

https://doi.org/10.1371/journal.pone.0009282 (2010).

46. Pimenta, F. C. et al. Sequential triplex real-time PCR assay for detecting 21 pneumococcal capsular serotypes that account for a high global disease burden. J. Clin. Microbiol, 51, 647-652 (2013).

47. Sakai, F. et al. Single-plex quantitative assays for the detection and quantification of most pneumococcal serotypes. PLoS One, 10, e0121064 https://doi.org/10.1371/journal.pone.0121064 (2015).

\section{Figures}

a

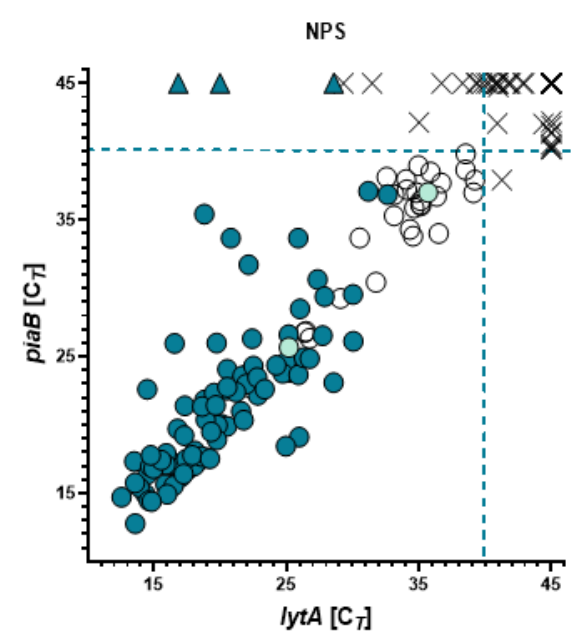

b

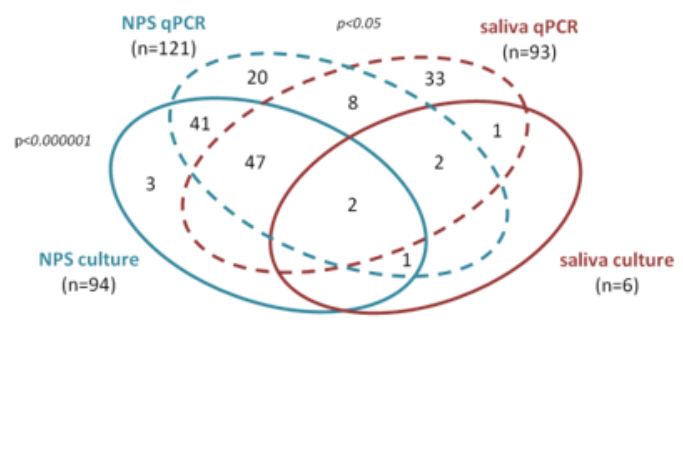

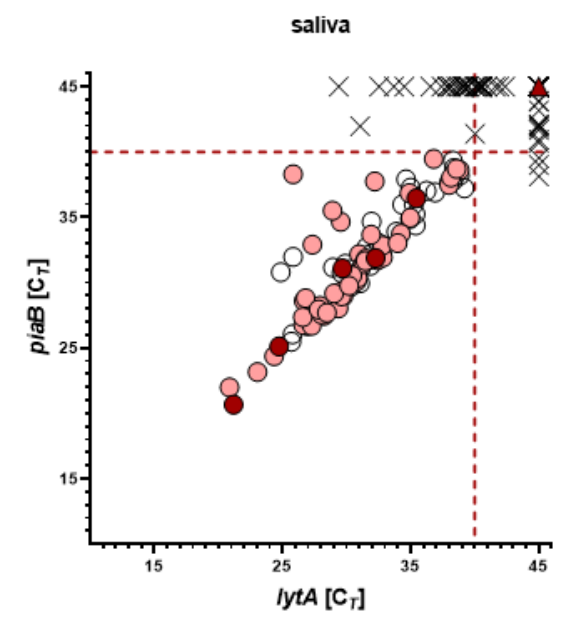

Figure 1

Detection of Streptococcus pneumoniae with molecular methods versus isolation of live pneumococci from nasopharyngeal (NPS) and saliva samples collected from 394 children. Left and right panels represent scatter plots of the lytA and piaB qPCR cycle threshold (CT) values from (a) NPS and (c) saliva samples. Venn diagram in panel (b) depicts the numbers of NPS and saliva samples positive for pneumococci based on recovery of live S. pneumoniae from a culture (solid-line ovals) or when tested with qPCRs (dashed-line ovals). Each symbol in panels (a) and (c) represents an individual sample. Samples with a CT for both piaB and lytA below 40 CT (marked with dashed lines) were considered positive for pneumococcus with molecular method. Dark-blue dots in panel (a), and dark-red dots in panel (c) represent NPS $(n=94)$ and saliva $(n=6)$ samples, respectively, from which live pneumococci have been isolated. Light-blue dots in panel $(a)(n=2)$, and light-red dots in panel $(c)(n=52)$ represent samples positive for $S$. pneumoniae by molecular method, negative by culture yet with live pneumococcus isolated from a paired sample (samples from culture-confirmed carriers). Open circles represent NPS ( $n=28)$, and saliva $(n=41)$ samples classified with molecular method as positive for $\mathrm{S}$. pneumoniae from children from whom no live $\mathrm{S}$. pneumoniae has been isolated. Triangles represent NPS $(n=3)$ and saliva $(n=1)$ samples negative by molecular method, from which live pneumococci have been isolated. Crosses represent NPS $(n=227)$, and saliva $(n=294)$ samples negative by any method used. The p-values reported in panel (b) were calculated with McNemar's test. 


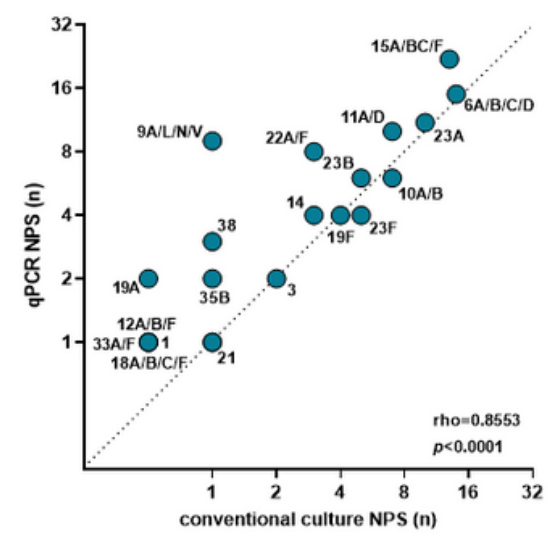

b

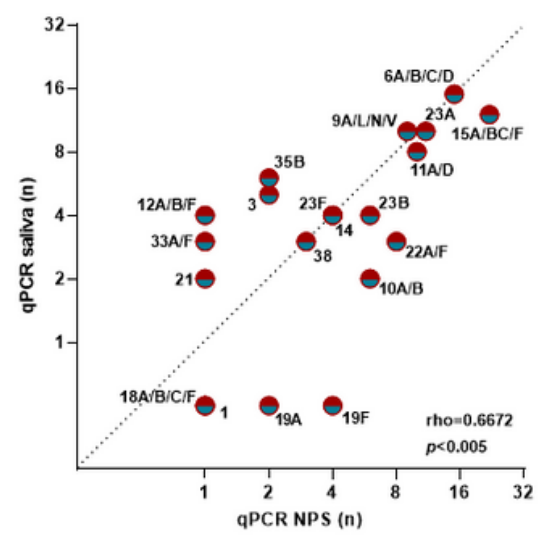

c

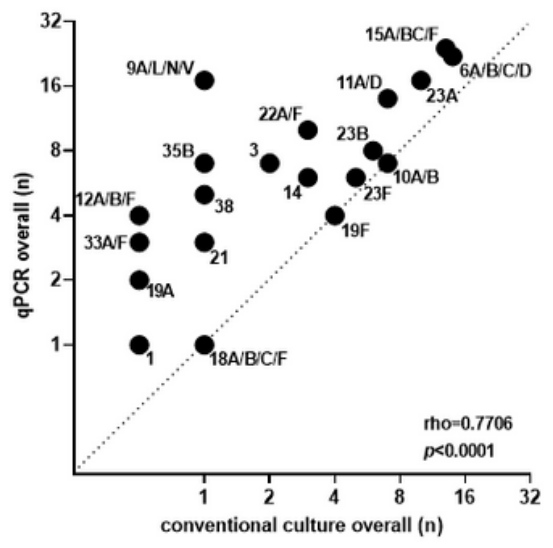

Figure 2

Correlations between results of pneumococcal serotypes detected by conventional culture and molecular method (qPCR). Panels (a) and (c) depict correlations between number of cultured (X-axis) and number of samples positive in qPCR among serotypes or serogroups targeted by qPCR assays. Panel (a) depicts results exclusively for NPS samples. Panel (c) depicts results for all strains cultured from NPS or saliva versus detected in NPS or saliva in qPCR assays. Panel (b) shows correlation between NPS (X-axis) and saliva (Y-axis) for serotypes detected exclusively with qPCR assays. Serotypes not detected using a given approach have been assigned a value of 0.5 . The rho, and $p$ values have been calculated with Spearman's test. 